\title{
Strongly stable algorithm for computing periodic system zeros
}

\author{
Andras Varga \\ German Aerospace Center \\ DLR - Oberpfaffenhofen \\ Institute of Robotics and System Dynamics \\ D-82234 Wessling, Germany \\ Andras.Varga@dlr.de
}

\begin{abstract}
We propose a computationally efficient and numerically reliable algorithm to compute the finite zeros of a linear discrete-time periodic system. The zeros are defined in terms of the transfer-function matrix corresponding to an equivalent lifted time-invariant state-space system. The proposed method relies on structure preserving manipulations of the associated system pencil to extract successively lower complexity subpencils which contains the finite zeros of the periodic system. The new algorithm uses exclusively structure preserving orthogonal transformations and for the overall computation of zeros the strong numerical stability can be proved.
\end{abstract}

\section{INTRODUCTION}

We consider the problem of computing zeros of periodic time-varying descriptor systems of the form

$$
\begin{aligned}
E_{k} x(k+1) & =A_{k} x(k)+B_{k} u(k) \\
y(k) & =C_{k} x(k)+D_{k} u(k)
\end{aligned}
$$

where the matrices $E_{k} \in \mathrm{R}^{\nu_{k+1} \times n_{k+1}}, A_{k} \in \mathrm{R}^{\nu_{k+1} \times n_{k}}$, $B_{k} \in \mathrm{R}^{\nu_{k+1} \times m_{k}}, C_{k} \in \mathrm{R}^{p_{k} \times n_{k}}, D_{k} \in \mathrm{R}^{p_{k} \times m_{k}}$ are periodic with period $K \geq 1$. For solvability of these equations we will assume that the dimensions of $A_{k}$ and $E_{k}$ fulfil the condition $\sum_{k=1}^{K} \nu_{k}=\sum_{k=1}^{K} n_{k}$.

A general, efficient and numerically reliable algorithm to compute the zeros of such a system represents a universal analysis tool of periodic systems. Besides characterizing when the system is minimum-phase or not, the zeros provide practically information on all structural properties of a system. For instance, reachability/stabilizability and observability/detectability can be easily studied by computing the zeros of particular periodic systems without outputs or inputs, respectively. Even the poles of a periodic system can be seen as a particular type of zeros for a system with no inputs and no outputs.

For the computation of zeros it is important to consider the more general case of time-varying dimensions. Since the transmission zeros of a standard system are defined in terms of a minimal realization, a similar definition is appropriate also for the zeros of a periodic system (see for example [10]). However, the minimal realization theory of standard periodic systems (i.e., $E_{k}=I_{n_{k+1}}$ ) revealed (see for example [3], [5]) that minimal order (i.e., reachable and observable) state-space realizations of periodic systems have, in general, time-varying state dimensions. It follows immediately that the minimal realization of a periodic descriptor system computed, for example, via a forward-backward decomposition
[14], leads in general to rectangular descriptor matrices $E_{k}$ as well. Time-varying input and output vector dimensions occur when evaluating zeros of particular system as those appearing in an algorithm to evaluate the transfer-function matrix of a periodic system [17]. Finally, the development of general algorithms able to address the case of time-varying dimensions, is one of the requirements formulated for a satisfactory numerical algorithm for periodic systems [18].

The definition of zeros of a periodic descriptor system can be introduced starting from two input-output equivalent time-invariant lifted reformulations [11], [8]. While the direct application of the numerically stable methods of [4], [12] to these representations is certainly possible, the resulting algorithms are completely inappropriate. Provided all $E_{k}$ are identity matrices (i.e., we have a standard periodic system), constructing explicitly the lifted representation of [11] involves forming products of up to $K$ matrices. Thus, applying the algorithm of [4] to this lifted system can lead to severe numerical difficulties. When applying the method of [12] to the large order lifted descriptor representation of [8], the computational complexity for large order systems or large periods is very high. Assuming constant dimensions $\mu_{i}=n_{i}=n$, such an algorithm has a complexity of $O\left(K^{3} n^{3}\right)$, instead of an expected complexity of $O\left(K n^{3}\right)$ for a satisfactory algorithm [18].

Specific requirements for satisfactory numerical algorithms for periodic systems have been formulated in [18]. Besides low computational complexity, the numerical stability of algorithms is a main requirement. A first general method to compute the zeros of periodic systems, belonging to the family of fast, structure exploiting algorithms, has been proposed in [19]. This algorithm relies exclusively on using orthogonal transformations and it can be shown that it is numerically stable in the following restricted sense: the computed zeros in the presence of roundoff errors are exact for a slightly perturbed lifted system pencil. However, by performing row compressions of the system pencil which destroy its cyclic structure, this algorithm is not strongly numerically stable. This means that it is not possible to demonstrate for it that the computed zeros are exact for an original system with slightly perturbed system matrices.

In this paper we propose a numerical approach to compute the finite zeros of the periodic system (1) which meets the requirements formulated in [18] for a satisfactory numerical 
algorithm regarding generality, speed and accuracy. The proposed method relies on structure preserving manipulations of the associated system pencil to extract successively lower complexity subpencils which contains the finite zeros of the periodic system. The new algorithm uses exclusively structure preserving orthogonal transformations and for the overall computation of zeros the strong numerical stability can be proved. Besides the finite zeros, the reduction algorithm also provides information to deduce the infinite zeros structure as well as the complete Kronecker structure of the lifted system pencil. For this reason, the proposed new algorithm can be seen as a generalization of the method of [12].

Notation. For a $K$-periodic matrix $X_{i} \in \mathrm{R}^{\mu_{k+1} \times n_{k}}$ we use alternatively the script notation

$$
\mathcal{X}_{k}:=\operatorname{diag}\left(X_{k}, X_{k+1}, \ldots, X_{k+K-1}\right),
$$

which associates the block-diagonal matrix $\mathcal{X}_{k}$ to the cyclic matrix sequence $X_{i}, i=k, \ldots, k+K-1$ starting at time moment $k$. We will use the notation $\mathbf{n}_{k}:=\left\{n_{k}, \ldots, n_{k+K-1}\right\}$ to denote the time-varying dimensions of periodic matrices. By using the script notation, the periodic system (1) will be alternatively denoted by the quintuple $\left(\mathcal{E}_{k}, \mathcal{A}_{k}, \mathcal{B}_{k}, \mathcal{C}_{k}, \mathcal{D}_{k}\right)$. The dimensional information on state-, input- and outputvectors of this system is provided by the triple $\left(\mathbf{n}_{k}, \mathbf{m}_{k}, \mathbf{p}_{k}\right)$. To simplify the notation for the case $k=1$, we drop the index used for the sampling time in the system matrices and dimensions.

\section{ZEROS AND POLES OF PERIODIC SYSTEMS}

We define the zeros and poles of the periodic system (1), using the transfer-function matrix (TFM) corresponding to the associated stacked lifted representation [8]. This lifting technique uses the input-output behavior of the system over time intervals of length $K$, rather then 1 . For a given sampling time $k$, the corresponding input-, output- and statevectors

$$
\begin{aligned}
u_{k}^{S}(h) & =\left[u^{T}(k+h K) \cdots u^{T}(k+h K+K-1)\right]^{T} \\
y_{k}^{S}(h) & =\left[y^{T}(k+h K) \cdots y^{T}(k+h K+K-1)\right]^{T} \\
x_{k}^{S}(h) & =\left[x^{T}(k+h K) \cdots x^{T}(k+h K+K-1)\right]^{T}
\end{aligned}
$$

have dimensions $M=\sum_{k=1}^{K} m_{k}, P=\sum_{k=1}^{K} p_{k}$ and $N=$ $\sum_{k=1}^{K} n_{k}$, respectively. The lifted system has a time-invariant descriptor system representation of the form

$$
\begin{aligned}
L_{k}^{S} x_{k}^{S}(h+1) & =F_{k}^{S} x_{k}^{S}(h)+G_{k}^{S} u_{k}^{S}(h) \\
y_{k}^{S}(h) & =H_{k}^{S} x_{k}^{S}(h)+J_{k}^{S} u_{k}^{S}(h)
\end{aligned}
$$

where $G_{k}^{S}=\mathcal{B}_{k}, H_{k}^{S}=\mathcal{C}_{k}, J_{k}^{S}=\mathcal{D}_{k}$, and

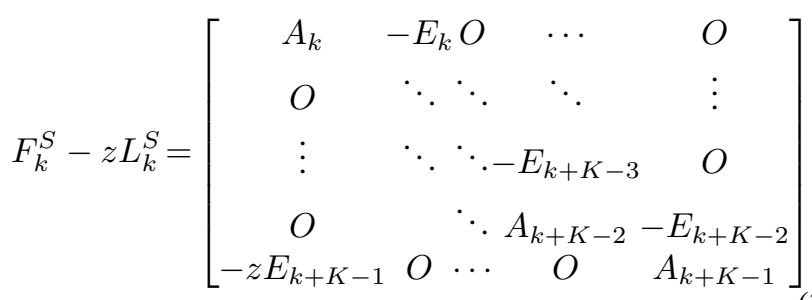

Assuming the square pencil (3) is regular (i.e. $\operatorname{det}\left(F_{k}^{S}-z L_{k}^{S}\right)$ is not identically 0 ), the TFM of the stacked lifted system is

$$
W_{k}(z)=H_{k}^{S}\left(z L_{k}^{S}-F_{k}^{S}\right)^{-1} G_{k}^{S}+J_{k}^{S}
$$

and the associated system pencil is defined as

$$
S_{k}^{S}(z)=\left[\begin{array}{cc}
F_{k}^{S}-z L_{k}^{S} & G_{k}^{S} \\
H_{k}^{S} & J_{k}^{S}
\end{array}\right],
$$

which both depend on the sampling time $k$. Obviously $W_{k+K}(z)=W_{k}(z)$ and the TFMs at two successive values of $k$ are related by the following relation [6]

$$
W_{k+1}(z)=\left[\begin{array}{cc}
0 & I_{P-p_{k}} \\
z I_{p_{k}} & 0
\end{array}\right] W_{k}(z)\left[\begin{array}{cc}
0 & z^{-1} I_{m_{k}} \\
I_{M-m_{k}} & 0
\end{array}\right] .
$$

It follows from this relation that poles and zeros of the TFMs for different sampling time, can only differ at $z=0$ and $z=\infty$. The normal rank $L$ of the TFM $W_{k}(z)$ (i.e., the rank over rationals) is the number of non-zero diagonal elements in the Smith-McMillan form of $W_{k}(z)$.

In order to define poles and zeros of the periodic system (1), we need the minimality of the system and of the realization (2). This is equivalent to the notion of reachability and observability at finite and infinite eigenvalues of the pencil (5), as introduced in [20]. If we assume that the system (1) is minimal in that sense (this implies time-varying state dimensions and rectangular descriptor matrices) then we have the following definitions of poles, zeros and minimal indices of the TFM (4) based on the system matrix (5) of the stacked lifted system (2).

Definition 1: The transmission zeros of the TFM $W_{k}(z)$ of the minimal order periodic system $\left(\mathcal{E}_{k}, \mathcal{A}_{k}, \mathcal{B}_{k}, \mathcal{C}_{k}, \mathcal{D}_{k}\right)$ are the invariant zeros of the associated system pencil (5).

Definition 2: The left and right minimal indices of the TFM $W_{k}(z)$ of the minimal periodic system $\left(\mathcal{E}_{k}, \mathcal{A}_{k}, \mathcal{B}_{k}, \mathcal{C}_{k}, \mathcal{D}_{k}\right)$ are those of the associated system pencil (5).

Definition 3: The poles of the TFM $W_{k}(z)$ of the minimal periodic system $\left(\mathcal{E}_{k}, \mathcal{A}_{k}, \mathcal{B}_{k}, \mathcal{C}_{k}, \mathcal{D}_{k}\right)$ are the zeros of the associated pole pencil $F_{k}^{S}-z L_{k}^{S}$ defined in (3).

The above definitions of zeros and poles of a periodic system are consistent with definitions based on the lifting technique introduced in [11] applicable to systems with $E_{k}$ square and invertible. In this case, the transfer-function matrices of the two lifted systems are the same, thus the corresponding definitions of poles and zeros coincide.

From the definition of zeros follows that the transmission zeros of the periodic system (1) (finite and infinite) are those values of $z$ where the rank of the lifted system matrix $S_{k}^{S}(z)$ drops below its normal rank $N+L$. The "infinite zeros" and their multiplicities can be defined in terms of the "infinite eigenvalues" of the pencil $S_{k}^{S}(z)$ (see the relationship between the null zeros of $W_{k}(1 / \lambda)$ and the Kronecker form of $S_{k}^{S}(z)$ [20]). To each Jordan block of size $j$ at the eigenvalue 
$\infty$ corresponds an elementary divisor $\lambda^{j-1}$ in the SmithMcMillan form of $S_{k}^{S}(1 / \lambda)$. Because of this difference of one for the structure at infinity, the simple eigenvalues at $\infty$ of the pencil $F_{k}^{S}-z L_{k}^{S}$ (at most $N-$ rank $E_{k+K-1}$ ) play no role when counting the infinite zeros.

\section{COMPUTATIONAL APPROACH}

In this section we propose an efficient computational approach to determine the zeros of the stacked lifted system (2) at $k=1$. The zeros for other time moments $k=2, \ldots, K$ can be computed in a similar manner by just permuting the order of the underlying matrices.

Instead of $S^{S}(z)$ in (5), we consider an equivalent pencil $S(z)$ with permuted block rows and columns

$$
S(z)=\left[\begin{array}{ccccc}
S_{1} & -T_{1} & O & \cdots & O \\
O & S_{2} & -T_{2} & \cdots & O \\
\vdots & \ddots & \ddots & \ddots & \vdots \\
O & & & S_{K-1} & -T_{K-1} \\
-z T_{K} & O & \cdots & O & S_{K}
\end{array}\right]
$$

where for $k=1, \ldots, K$

$$
S_{k}:=\left[\begin{array}{cc}
A_{k} & B_{k} \\
C_{k} & D_{k}
\end{array}\right], \quad T_{k}:=\left[\begin{array}{cc}
E_{k} & O \\
O & O
\end{array}\right] .
$$

To reduce this pencil by preserving its structure, we will use orthogonal transformations of the form

$$
\widetilde{S}_{k}=Q_{k} S_{k} Z_{k}, \quad \widetilde{T}_{k}=Q_{k} T_{k} Z_{k+1}
$$

which corresponds to apply to $S(z)$ from left and right, the block-diagonal matrices $\mathcal{Q}$ and $\mathcal{Z}$, respectively.

The proposed algorithm to compute zeros can be applied to compute the system poles as well by defining

$$
S_{k}:=A_{k}, \quad T_{k}:=E_{k} .
$$

In a similar way, with

$$
S_{k}:=\left[\begin{array}{ll}
A_{k} & B_{k}
\end{array}\right], \quad T_{k}:=\left[\begin{array}{ll}
E_{k} & O
\end{array}\right]
$$

or

$$
S_{k}:=\left[\begin{array}{c}
A_{k} \\
C_{k}
\end{array}\right], \quad T_{k}:=\left[\begin{array}{c}
E_{k} \\
O
\end{array}\right]
$$

the zeros algorithm can be used to compute the input decoupling zeros and output decoupling zeros, respectively [7]. We also discuss some computational enhancements which arise in the case of standard periodic systems (i.e., $E_{k}=I_{n_{k+1}}$ ). The algorithm we propose has three main steps, which we discuss in the subsequent three subsections.

\section{A. Computation of the compressed system}

In the first step we reduce the problem to an equivalent one, but for square and non-singular periodic descriptor matrices. Let $\mathcal{U}$ and $\mathcal{V}$ be orthogonal periodic matrices such that

$$
\begin{gathered}
{\left[\begin{array}{c|c}
U_{k} & O \\
\hline O & I
\end{array}\right]\left[\begin{array}{c|c}
A_{k} & B_{k} \\
\hline C_{k} & D_{k}
\end{array}\right]\left[\begin{array}{c|c}
V_{k} & O \\
\hline O & I
\end{array}\right]=\left[\begin{array}{c:c|c}
A_{k, 11} & A_{k, 12} & B_{k, 1} \\
\hdashline A_{k, 21} & A_{k, 22} & B_{k, 2} \\
\hline C_{k, 1} & C_{k, 2} & D_{k}
\end{array}\right]} \\
{\left[\begin{array}{c|c}
U_{k} & O \\
\hline O & I
\end{array}\right]\left[\begin{array}{c|c|c}
E_{k} & O \\
\hline O & O
\end{array}\right]\left[\begin{array}{c|c|c}
V_{k+1} & O \\
\hline O & I
\end{array}\right]=\left[\begin{array}{c:c|c}
E_{k, 11} & O & O \\
\hdashline O & O & O \\
\hline O & O & O
\end{array}\right]}
\end{gathered}
$$

where $E_{k, 11} \in \mathrm{R}^{r_{k+1} \times r_{k+1}}$ for $k=1, \ldots, K$, are square, non-singular matrices. The compression of each $E_{k}$ to a nonsingular $E_{k, 11}$ can be done by computing a full orthogonal decomposition $U_{k} E_{k} V_{k+1}=\operatorname{diag}\left(E_{k, 11}, O\right)$ using either the singular-value decomposition (SVD) or a rank-revealing $\mathrm{QR}$ decomposition followed by an RQ-decomposition. In both cases, we can assume that each $E_{k, 11}$ results upper triangular.

If we construct the new system matrices

$$
\begin{gathered}
\bar{E}_{k}=E_{k, 11}, \quad \bar{A}_{k}=A_{k, 11}, \quad \bar{B}_{k}=\left[\begin{array}{cc}
A_{k, 12} & B_{k, 1}
\end{array}\right] \\
\bar{C}_{k}=\left[\begin{array}{c}
A_{k, 21} \\
C_{k, 1}
\end{array}\right], \quad \bar{D}_{k}=\left[\begin{array}{cc}
A_{k, 22} & B_{k, 2} \\
C_{k, 2} & D_{k}
\end{array}\right]
\end{gathered}
$$

then the pencils $S(z)$ and the transformed pencil $\bar{S}(z)$ corresponding to the reduced matrices have the same Kroneckercanonical form, thus we have the following straightforward result.

Theorem 1: The original system $(\mathcal{E}, \mathcal{A}, \mathcal{B}, \mathcal{C}, \mathcal{D})$ and the "compressed system" $(\overline{\mathcal{E}}, \overline{\mathcal{A}}, \overline{\mathcal{B}}, \overline{\mathcal{C}}, \overline{\mathcal{D}})$ have the same transmission zeros, left and right minimal indices.

Note that in general the "compressed system" has timevarying dimensions not only for the state vector but also for the input and output vectors, even when the original system has constant input and output dimensions.

\section{B. Isolation of the finite part}

In the second step we isolate a periodic descriptor system $\left(\mathcal{E}^{r c}, \mathcal{A}^{r c}, \mathcal{B}^{r c}, \mathcal{C}^{r c}, \mathcal{D}^{r c}\right)$ where $\mathcal{E}^{r c}$ and $\mathcal{D}^{r c}$ are square invertible matrices. Once reduced to this form, the transmission zeros of the system are the characteristic multipliers of the periodic pair $\left(\mathcal{E}^{r c}, \mathcal{A}^{r c}-\mathcal{B}^{r c}\left(\mathcal{D}^{r c}\right)^{-1} \mathcal{C}^{r c}\right)$. It will be shown later that the characteristic multipliers can be obtained without inverting $\mathcal{D}^{r c}$.

The goal of the reduction in this step is to isolate a regular part of the transformed pencil $\bar{S}(z)$ which contains the finite zeros. For this purpose we redefine the dimensions $m_{k}:=$ $n_{k}-r_{k}+m_{k}, p_{k}:=\nu_{k+1}-r_{k+1}+p_{k}$ and $n_{k}:=r_{k}$. For convenience, we will also reuse the original notation by redefining $(\mathcal{E}, \mathcal{A}, \mathcal{B}, \mathcal{C}, \mathcal{D}):=(\overline{\mathcal{E}}, \overline{\mathcal{A}}, \overline{\mathcal{B}}, \overline{\mathcal{C}}, \overline{\mathcal{D}})$.

The isolation of the part containing the finite zeros is done in two steps. In the first step, we isolate a part which corresponds to a periodic descriptor system $\left(\mathcal{E}^{r}, \mathcal{A}^{r}, \mathcal{B}^{r}, \mathcal{C}^{r}, \mathcal{D}^{r}\right)$, where $\mathcal{E}^{r}$ is square invertible and $\mathcal{D}^{r}$ is full row rank. This is performed by employing the following procedure generalizing the Algorithm S-REDUCE of [12]: 


\section{Algorithm PS-REDUCE}

input $(\mathcal{E}, \mathcal{A}, \mathcal{B}, \mathcal{C}, \mathcal{D}, \mathbf{n}, \mathbf{m}, \mathbf{p})$, output $\left(\mathcal{E}^{r}, \mathcal{A}^{r}, \mathcal{B}^{r}, \mathcal{C}^{r}, \mathcal{D}^{r}, \mathbf{n}^{\mathbf{r}}, \mathbf{m}^{\mathbf{r}}, \mathbf{p}^{\mathbf{r}}\right)$.

step-i

1. For each $k=1, \ldots, K$, compress the rows of $D_{k}$ with orthogonal $X_{k}^{(i)}$ and transform $C_{k}$;

$$
\left[\begin{array}{c|c}
C_{k, 1} & D_{k, 1} \\
C_{k, 2} & O
\end{array}\right]:=X_{k}^{(i)}\left[C_{k} \mid D_{k}\right],
$$

where $D_{k, 1} \in \mathrm{R}^{\left(p_{k}-\tau_{k}^{(i)}\right) \times m_{k}}$ has full row rank and $C_{k, 2} \in \mathrm{R}^{\tau_{k}^{(i)} \times n_{k}}$; if $\tau_{k}^{(i)}=0$ for $k=1, \ldots, K$, then go to exit-1

2. For each $k=1, \ldots, K$, compress the columns of $C_{k, 2}$ with orthogonal $V_{k}^{(i)}$ such that $C_{k, 2} V_{k}^{(i)}=\left[\begin{array}{ll}O & C_{k, 22}\end{array}\right]$, with $C_{k, 22} \in \mathrm{R}^{\tau_{k}^{(i)} \times \mu_{k}^{(i)}}$ full column rank;

3. For each $k=1, \ldots, K$, determine orthogonal $U_{k}^{(i)}$ such that $U_{k}^{(i)} E_{k} V_{k+1}^{(i)}$ is upper triangular; transform the system and partition as:

$$
\begin{gathered}
{\left[\begin{array}{c|c}
U_{k}^{(i)} & O \\
\hline O & X_{k}^{(i)}
\end{array}\right]\left[\begin{array}{c|c}
A_{k} & B_{k} \\
\hline C_{k} & D_{k}
\end{array}\right]\left[\begin{array}{c|c}
V_{k}^{(i)} & O \\
\hline O & I_{m_{k}}
\end{array}\right]} \\
=\left[\begin{array}{c:c|c}
A_{k, 11} & A_{k, 12} & B_{k, 1} \\
\hdashline A_{k, 21} & A_{k, 22} & B_{k, 2} \\
\hline C_{k, 11} & C_{k, 12} & D_{k, 1} \\
\hdashline O & C_{k, 22} & O
\end{array}\right] \\
U_{k}^{(i)} E_{k} V_{k+1}^{(i)}=\left[\begin{array}{c:c}
E_{k, 11} & E_{k, 12} \\
\hdashline O & E_{k, 22}
\end{array}\right],
\end{gathered}
$$

where $A_{k, 22} \in \mathrm{R}^{\mu_{k+1}^{(i)} \times \mu_{k}^{(i)}}$.

4. Set $A_{k}:=A_{k, 11}, E_{k}:=E_{k, 11}, B_{k}:=B_{k, 1}, C_{k}:=$ $\left[\begin{array}{l}A_{k, 21} \\ C_{k, 11}\end{array}\right], D_{k}:=\left[\begin{array}{c}B_{k, 2} \\ D_{k, 1}\end{array}\right]$.

5. Update $n_{k}:=n_{k}-\mu_{k}^{(i)}, p_{k}:=p_{k}-\left(\tau_{k}^{(i)}-\mu_{k+1}^{(i)}\right)$, for $k=1, \ldots, K$.

6. If $n_{k}=0$ for $k=1, \ldots, K$, then go to exit-2

7. If $\mu_{k}^{(i)}=0$ for $k=1, \ldots, K$, then go to exit-1

8. $i:=i+1$ go to step-i;

exit-1 comment Full rank matrix $\mathcal{D}^{r}$ found.

$\left(\mathcal{E}^{r}, \mathcal{A}^{r}, \mathcal{B}^{r}, \mathcal{C}^{r}, \mathcal{D}^{r}\right):=(\mathcal{E}, \mathcal{A}, \mathcal{B}, \mathcal{C}, \mathcal{D})$

$\mathbf{n}^{\mathbf{r}}:=\mathbf{n} ; \mathbf{m}^{\mathbf{r}}:=\mathbf{m} ; \mathbf{p}^{\mathbf{r}}:=\mathbf{p}$.

exit-2 comment System has no finite zeros.

Remark. The compression of $C_{k, 2}$ to a full column rank matrix can be done simultaneously with maintaining $E_{k}$ upper triangular by using an algorithm similar to that of [15] for standard descriptor systems. Details for achieving this are given in the next section. Obvious simplifications arise when $E_{k}=I$. In this case, it possible to devise PS-REDUCE such that the reduced $E_{k}^{r}$ is also the identity matrix. This amounts to reduce $C_{k, 2}$ by performing Lyapunov similarity transformations, thus ensuring that $U_{k}^{(i)}=V_{k+1}^{(i)}$.
The PS-REDUCE algorithm determines implicitly the normal rank of the TFM $W(z)$. If $\rho_{K}$ is the rank defect of the original descriptor matrix $E_{K}$, then the normal rank of the TFM $W_{k}(z)$ is

$$
L=\sum_{k=1}^{K} p_{k}^{r}-\rho_{K}
$$

At the end of PS-REDUCE algorithm we obtain globally the reduced matrices $\widetilde{S}_{k}$ and $\widetilde{T}_{k}$ in (7) in the form

$$
\widetilde{S}_{k}=\left[\begin{array}{cc|c}
A_{k}^{r} & B_{k}^{r} & * \\
C_{k}^{r} & D_{k}^{r} & * \\
\hline O & O & S_{k}^{r}
\end{array}\right], \quad \widetilde{T}_{k}=\left[\begin{array}{cc|c}
E_{k}^{r} & O & * \\
O & O & * \\
\hline O & O & T_{k}^{\bar{r}}
\end{array}\right]
$$

where each $S_{k}^{\bar{r}}$ has full column rank and the leading nonzero rows of $T_{k}^{\bar{r}}$ form a full row rank matrix. The overall reduced system pencil can be put, after obvious row and column permutations, in the form

$$
\widehat{S}(z)=\left[\begin{array}{cc}
S^{r}(z) & * \\
O & S^{\bar{r}}(z)
\end{array}\right],
$$

with $S^{x}(z)(x=r$ or $\bar{r})$ of the form

$$
S^{x}(z)=\left[\begin{array}{ccccc}
S_{1}^{x} & -T_{1}^{x} & O & \cdots & O \\
O & S_{2}^{x} & -T_{2}^{x} & \cdots & O \\
\vdots & \ddots & \ddots & \ddots & \vdots \\
O & & & S_{K-1}^{x} & -T_{K-1}^{x} \\
-z T_{K}^{x} & O & \cdots & O & S_{K}^{x}
\end{array}\right]
$$

where for $k=1, \ldots, K$

$$
\begin{aligned}
& S_{k}^{r}:=\left[\begin{array}{cc}
A_{k}^{r} & B_{k}^{r} \\
C_{k}^{r} & D_{k}^{r}
\end{array}\right], \quad T_{k}^{r}:=\left[\begin{array}{cc}
E_{k}^{r} & O \\
O & O
\end{array}\right] . \\
& S_{k}^{\bar{r}}=\left[\begin{array}{ccccc}
A_{k ; \ell, \ell}^{\bar{r}} & A_{k ; \ell, \ell-1}^{\bar{r}} & \cdots & A_{k ; \ell, 2}^{\bar{r}} & A_{k ; \ell, 1}^{\bar{r}} \\
O & A_{k ; \ell-1, \ell-1}^{\bar{r}} & \cdots & A_{k ; \ell, 2}^{\bar{r}} & A_{k ; \ell, 1}^{\bar{r}} \\
\vdots & \vdots & \ddots & \vdots & \vdots \\
O & O & \cdots & A_{k ; 2,2}^{\bar{r}} & A_{k ; 2,1}^{\bar{r}} \\
O & O & \cdots & O & A_{k ; 1,1}^{\bar{r}}
\end{array}\right] \\
& T_{k}^{\bar{r}}=\left[\begin{array}{ccccc}
O & E_{k ; \ell, \ell-1}^{\bar{r}} & \cdots & E_{k ;}^{\bar{r}} & E_{k ; \ell, 2}^{\bar{r}} \\
O & O & \cdots & E_{k ; \ell, 2}^{\bar{r}} & E_{k ; \ell, 1}^{\bar{r}} \\
\vdots & \vdots & \ddots & \vdots & \vdots \\
O & O & \cdots & O & E_{k ; 2,1}^{\bar{r}} \\
O & O & \cdots & O & O
\end{array}\right]
\end{aligned}
$$

where $\ell$ is the number of steps performed in the Algorithm PS-REDUCE, $A_{k ; i, i}^{\bar{r}} \in \mathrm{R}^{\left.\tau_{k}^{(i)} \times \mu_{k}^{(i)}\right)}$ is full column rank and $E_{k ; i, i-1}^{\bar{r}} \in \mathrm{R}^{\tau_{k}^{(i+1)} \times \mu_{k+1}^{(i)}}$ is full row rank. The subpencils $S^{r}(z)$ and $S^{\bar{r}}(z)$ have the same structure as the original system pencil $S(z)$. $S^{r}(z)$ contains the finite zeros of the periodic system and the information on the right Kronecker structure (e.g., right nullspace) of $S(z)$. The trailing subpencil $S^{\bar{r}}(z)$, has full column rank for all finite values of $z$, an thus contains information on the orders of infinite zeros and 
the left Kronecker structure (e.g., left nullspace) of $S(z)$. These facts can be proven similarly as done in [13] for standard systems. This leads to the following result.

Theorem 2: The orders of the infinite elementary divisors of $S^{\bar{r}}(z)$ are equal to the orders of the infinite zeros of the system $(\mathcal{E}, \mathcal{A}, \mathcal{B}, \mathcal{C}, \mathcal{D})$.

A dual algorithm to PS-REDUCE can be devised to compute a reduced system $\left(\mathcal{E}^{c}, \mathcal{A}^{c}, \mathcal{B}^{c}, \mathcal{C}^{c}, \mathcal{D}^{c}\right)$, where $\mathcal{E}^{c}$ is square invertible and $\mathcal{D}^{c}$ is full column rank. In this case, the overall reduced system pencil can be put, after row and column permutations, in the form

$$
\widehat{S}(z)=\left[\begin{array}{cc}
S^{\bar{c}}(z) & * \\
O & S^{c}(z)
\end{array}\right]
$$

where both $S^{c}(z)$ and $S^{\bar{c}}(z)$ have the same structure as $S(z)$. This time $S^{c}(z)$ contains the finite and left Kronecker structure, while $S^{\bar{c}}(z)$, having full row rank for all finite $z$, contains the infinite and right Kronecker structure.

By performing these two algorithms successively, we get finally $\left(\mathcal{E}^{r c}, \mathcal{A}^{r c}, \mathcal{B}^{r c}, \mathcal{C}^{r c}, \mathcal{D}^{r c}\right)$, the reduced system with both $\mathcal{E}^{r c}$ and $\mathcal{D}^{r c}$ square invertible matrices. Overall we can then show the following result.

Theorem 3: The system $(\mathcal{E}, \mathcal{A}, \mathcal{B}, \mathcal{C}, \mathcal{D})$ and the reduced system $\left(\mathcal{E}^{r c}, \mathcal{A}^{r c}, \mathcal{B}^{r c}, \mathcal{C}^{r c}, \mathcal{D}^{r c}\right)$ have the same finite transmission zeros.

As already mentioned, the finite zeros can be computed as the characteristic multipliers of the periodic pair $\left(\mathcal{E}^{r c}, \mathcal{A}^{r c}-\mathcal{B}^{r c}\left(\mathcal{D}^{r c}\right)^{-1} \mathcal{C}^{r c}\right)$, where $\mathcal{E}^{r c}$ is invertible. To avoid the inversion of $\mathcal{D}^{r c}$, it is possible to deflate a part of simple infinite (non-dynamic) eigenvalues by performing a final compression of the system matrices. To do that, we determine $V_{k}$ for $k=1, \ldots, K$ by compressing the columns of $\left[\begin{array}{ll}C_{k}^{r c} & D_{k}^{r c}\end{array}\right]$ to $\left[\begin{array}{ll}O & D_{k}^{f}\end{array}\right]$ and transforming the reduced system matrices as

$$
\begin{aligned}
& {\left[\begin{array}{c|c}
A_{k}^{f} & * \\
\hline O & D_{k}^{f}
\end{array}\right]:=\left[\begin{array}{cc}
A_{k}^{r c} & B_{k}^{r c} \\
C_{k}^{r c} & D_{k}^{r c}
\end{array}\right] V_{k},} \\
& {\left[\begin{array}{c|c}
E_{k}^{f} & * \\
\hline O & O
\end{array}\right]:=\left[\begin{array}{cc}
E_{k}^{r c} & O \\
O & O
\end{array}\right] V_{k+1} .}
\end{aligned}
$$

This reduction can be always performed such that the resulting non-singular $E_{k}^{f}$ are upper triangular (see [1, pp. 33-34]).

By this final reduction we succeeded to isolate the regular part $S^{f}(z)$ of the system pencil $S(z)$ which contains the finite transmission zeros. This part has the same structure as the original system pencil, thus we can freely associate this pencil to a periodic eigenvalue problem defined by the periodic pair $\left(\mathcal{E}^{f}, \mathcal{A}^{f}\right)$. The characteristic multipliers of this pair are the finite transmission zeros of the periodic system.

\section{Computation of the finite zeros}

The third step of zeros computations consists in solving the periodic eigenvalue problem for the resulting periodic pair $\left(\mathcal{E}^{f}, \mathcal{A}^{f}\right)$. For constant dimension, the periodic QZ-algorithm
[2], [9] can be employed for this purpose. For time-varying dimensions the extended periodic Schur form based reduction [16] can be applied to the $n_{k+1}^{f} \times n_{k}^{f}$ periodic matrices $\left(E_{k}^{f}\right)^{-1} A_{k}^{f}, k=1, \ldots, K$. This is always possible, since $E_{k}^{f}$ is non-singular (and also upper triangular). However, a strongly numerically stable approach must avoid any inversion, and therefore we can easily extend the approach of [16] to compute two periodic transformation matrices $U_{k}$ and $V_{k}$ such that

$$
\begin{aligned}
& \widetilde{A}_{k}^{f}:=U_{k} A_{k}^{f} V_{k}=\left[\begin{array}{cc}
A_{k, 11}^{f} & A_{k, 12}^{f} \\
0 & \widetilde{A}_{k, 22}^{f}
\end{array}\right], \\
& \widetilde{E}_{k}^{f}:=U_{k} E_{k}^{f} V_{k+1}=\left[\begin{array}{cc}
E_{k, 11}^{f} & E_{k, 12}^{f} \\
0 & \widetilde{E}_{k, 22}^{f}
\end{array}\right],
\end{aligned}
$$

where $A_{k, 11}^{f}, E_{k, 11}^{f} \in \mathrm{R} \underline{n}^{f} \times \underline{n}^{f}$ for $\underline{n}^{f}=\min _{k}\left\{n_{k}^{f}\right\}, A_{k, 22}^{f}, \in$ $\mathrm{R}^{\left(n_{k+1}^{f}-\underline{n}^{f}\right) \times\left(n_{k}^{f}-\underline{n}^{f}\right)}, E_{k, 22}^{f}, \in \mathrm{R}^{\left(n_{k+1}^{f}-\underline{n}^{f}\right) \times\left(n_{k+1}^{f}-\underline{n}^{f}\right)}$ for $k=1, \ldots, K$. Moreover, one of matrices in the leading position, say $A_{K, 11}^{f}$, is in Hessenberg form, $A_{k, 11}^{f}$ for $k=$ $1 \ldots, K-1, E_{k, 11}^{f}$ and $E_{k, 22}^{f}$ for $k=1 \ldots, K$ are upper triangular, and $A_{k, 22}$ for $k=1 \ldots, K$ are upper trapezoidal. Thus, the pair $\left(\mathcal{E}_{11}^{f}, \mathcal{A}_{11}^{f}\right)$ is in a generalized periodic Hessenberg form as required by the application of the periodic QZalgorithm. By applying this algorithm we compute $\underline{n}_{f}$ finite zeros representing the so-called core set of zeros, which are independent of the time moment $k$. Additionally, there are also $n_{1}^{f}-\underline{n}^{f}$ null zeros, whose number varies according to the chosen time moment $k$.

\section{NUMERICAL ASPECTS}

For the reduction of $S(z)$ we employed exclusively structure preserving orthogonal transformations of the form (7). Thus it possible to prove that the computed finite zeros are exact for slightly perturbed initial matrices $\bar{S}_{k}, \bar{T}_{k}$, which satisfy

$$
\|\bar{X}-X\| \leq \varepsilon_{X}\|X\|, \quad X=S_{k}, T_{k}
$$

where, in each case, $\varepsilon_{X}$ is a modest multiple of the relative machine precision $\varepsilon_{M}$. It follows that the proposed algorithm is strongly backward stable.

Regarding the computational complexity of the proposed algorithm, we note that all reductions are performed $K$ times on low order matrices, thus the overall computational complexity is proportional with $K$. To estimate the worstcase computational complexity, we assume constant dimensions $n, m$ and $p$ for state-, input- and output vectors, respectively, and $E_{k}$ are invertible. The "system compression" performed by using either SVD-based or rank-revealing QRdecomposition based reductions requires $O\left(K n^{3}\right)$ floating point operations (flops). The compressions of $D_{k}, k=$ $1, \ldots, K$ can be done by computing successively $K$ rankrevealing QR-decompositions of $p \times m$ matrices and applying the transformation to a $p \times n$ sub-block. This reduction step, although performed more than once, has a computational 
complexity of $O(K(n+p) p n)$. A worst-case computational complexity of $O\left(K n^{3}\right)$ is also guaranteed for the last steps, to compute the finite zeros via the extended QZ-algorithm. The only critical computation is to maintain efficiently the upper triangular form of $E_{k}$ at successive steps of the PS-REDUCE algorithm. Note that by just computing $U_{k}$ such that $U_{k}^{(i)} E_{k} V_{k+1}^{(i)}$ is upper triangular is an operation of complexity $O\left(n^{3}\right)$. This would make the overall worst-case complexity to maintain $E_{k}$ upper triangular for $k=1, \ldots, K$ to become $O\left(K n^{4}\right)$. To avoid this, we can perform the compression of $C_{k, 2}$ with $V_{k}^{(i)}$ and restoring the upper-triangular form of $E_{k-1} V_{k}^{(i)}$ simultaneously, by employing Givens rotations. The technique is entirely similar to that proposed in [15] and also employed in [12]. Overall, this requires to perform the reduction for values of $k$ in a reverse order, for $k=K, K-1, \ldots, 1$. Using this approach, this computation has per iteration step a complexity at most $O\left(K \eta n^{2}\right)$, where $\eta$ is small compared to $n$. Thus, the overall complexity of the compression-restoring algorithm is $O\left(K n^{3}\right)$. Summing up, the computation of finite zeros has a worst-case complexity which can be bounded by $O K(p+n)(m+n) n$, which corresponds to what is expected for a satisfactory algorithm for periodic systems [18].

\section{CONCLUSION}

In this paper we developed a strongly numerically backward stable algorithm to compute the finite zeros of a stacked system matrix of a periodic system. This algorithm can be applied to find the finite zeros, finite poles and finite decoupling zeros of the system matrix and provides information to determine the orders of infinite zeros as well as the left and right nullspace structures of the corresponding lifted transfer function. These last aspects will be addressed in a separate paper. The algorithm works for matrices of varying dimension, and preserves the block cyclic structure of the corresponding lifted system pencil. This leads to two main benefits: 1) a satisfactory worst-case computational complexity, which is linear in the period $K$ and cubic in the maximum dimension of the blocks; and 2) strong numerical stability achieved by employing exclusively structure preserving orthogonal transformations. According to [18], this algorithm is well-suited for robust software implementations.

\section{REFERENCES}

[1] T. Beelen and P. Van Dooren. An improved algorithm for the computation of Kronecker's canonical form of a singular pencil. Lin. Alg. \& Appl., 105:9-65, 1988.

[2] A. W. Bojanczyk, G. Golub, and P. Van Dooren. The periodic Schur decomposition. Algorithms and applications. In F. T. Luk (Ed.), Proceedings SPIE Conference, vol. 1770, pp. 31-42, July 1992.

[3] P. Colaneri and S. Longhi. The realization problem for linear periodic systems. Automatica, 31:775-779, 1995.
[4] A. Emami-Naeini and P. M. Van Dooren. Computation of zeros of linear multivariable systems. Automatica, 18:415-430, 1982.

[5] I. Gohberg, M. A. Kaashoek, and J. Kos. Classification of linear periodic difference equations under periodic or kinematic similarity. SIAM J. Matrix Anal. Appl., 21:481-507, 1999.

[6] O. M. Grasselli and S. Longhi. Zeros and poles of linear periodic discrete-time systems. Circuits, Systems and Signal Processing, 7:361-380, 1988.

[7] O. M. Grasselli and S. Longhi. Finite zero structure of linear periodic discrete-time systems. Int. J. Systems Sci., 22:1785-1806, 1991.

[8] O. M. Grasselli and S. Longhi. Pole-placement for nonreachable periodic discrete-time systems. Math. Control Signals Syst., 4:439-455, 1991.

[9] J. J. Hench and A. J. Laub. Numerical solution of the discrete-time periodic Riccati equation. IEEE Trans. Autom. Control, 39:1197-1210, 1994.

[10] A. MacFarlane and N. Karcanias. Poles and zeros of linear multivariable systems: a survey of the algebraic, geometric, and complex-variable theory. Int. J. Control, 24:33-74, 1976.

[11] R. A. Meyer and C. S. Burrus. A unified analysis of multirate and periodically time-varying digital filters. IEEE Trans. Circuits and Systems, 22:162-168, 1975.

[12] P. Misra, P. Van Dooren, and A. Varga. Computation of structural invariants of generalized state-space systems. Automatica, 30:1921-1936, 1994.

[13] F. Svaricek. Computation of the structural invariants of linear multivariable systems with an extended version of the program ZEROS. Systems \& Control Lett., 6:261266, 1985.

[14] P. Van Dooren. Two point boundary value and periodic eigenvalue problems. Proc. CACSD'99 Symposium, Kohala Coast, Hawaii, 1999.

[15] A. Varga. Computation of irreducible generalized statespace realizations. Kybernetika, 26:89-106, 1990.

[16] A. Varga. Balancing related methods for minimal realization of periodic systems. Systems \& Control Lett., 36:339-349, 1999.

[17] A. Varga. Computation of transfer functions matrices of periodic systems. Proc. of CDC'2002, Las Vegas, Nevada, 2002.

[18] A. Varga and P. Van Dooren. Computational methods for periodic systems - an overview. Proc. of IFAC Workshop on Periodic Control Systems, Como, Italy, pp. 171-176, 2001.

[19] A. Varga and P. Van Dooren. Computing the zeros of periodic descriptor systems. Systems \& Control Lett., 50, 2003 (to appear).

[20] G. Verghese, P. Van Dooren, and T. Kailath. Properties of the system matrix of a generalized state-space system. Int. J. Control, 30:235-243, 1979. 